\title{
Modeling and Optimization for a Multi-degree Manipulator with Constrained Positions
}

\author{
Guojun $\mathrm{Yu}^{1, *}$, Yu Liu ${ }^{2}$ and Tiger Sun ${ }^{1}$ \\ ${ }^{1}$ Faculty of Civil Engineering and Mechanics, Jiangsu University, Zhenjiang, Jiangsu, China \\ ${ }^{2}$ Jiangsu Zhongsen Architectural Design Co., Ltd, Zhenjiang, Jiangsu, China \\ *Corresponding author
}

\begin{abstract}
For the urgent demand of lunar landing program, design a space manipulator and its arm with a flexible rotation, high precision, non-polluting and a vacuum operation from a long distance. Determining all the possible constrained positions and optimizing them by finite element software Abaqus, we get the effect factor of each constrained position and the order of the fracture under shock loading finally. Sincerely hope that this work can promote the space manipulator and high-speed development.
\end{abstract}

Keywords-manipulator; modeling; modular analysis; finite element method; optimization

\section{INTRODUCTION}

Along with the development of space station, space shuttle and space robot, the space manipulator has entered the space as in-orbit support, and services the new spacecraft [1-6]. So it has been pay close attention to the relevant scholars and scientists as shown in Figure 1 [7-10]. This work aims at the lunar landing program and designs a kind of multi-degree space manipulator and the arm supported the lunar landing detector, which is a flexible rotation, high precision, non-polluting and a vacuum operation from a long distance as shown in Figure 2. Considering the spatial constraints, we must optimize the fixed position of the space manipulator and the arm. First of all, it is necessary to choose all of possible fixed positions and to establish 3D model, then to calculate its intrinsic modes and static analysis, we get the effect factor of each constrained position and the order of the fracture under shock loading finally. We will obtain the better and optimized structure and last.

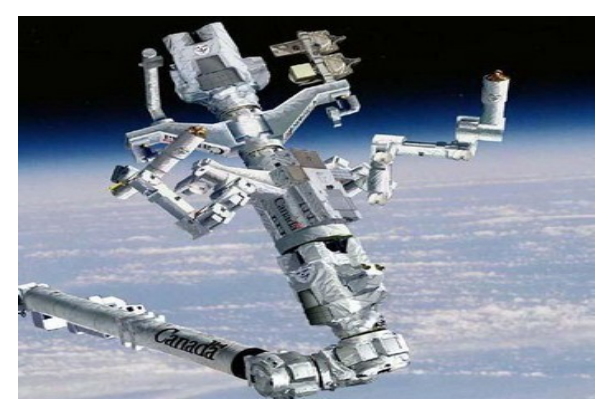

FIGURE I. THE WORKING SPACE MANIPULATOR

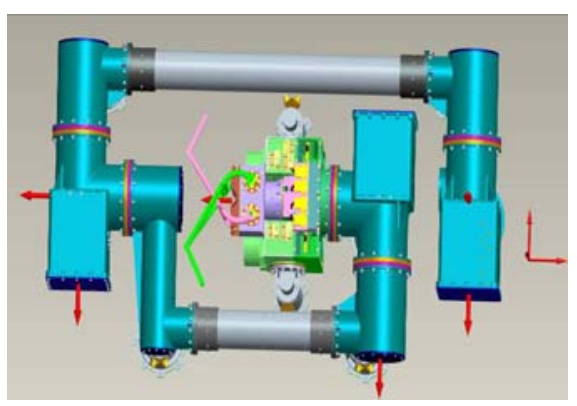

FIGURE II. 3D MODEL OF THE MANIPULATOR

\section{MECHANICS MODEL AND DyNAMiCs EQUATION}

Every joint of the space manipulator designed could be controlled accurately between 0-360 degrees in this 3D model. In order to improve the accuracy and range of their motion activity and considering its structure and incentive characteristics, their mechanical model must be abstracted as shown in Figure 3. Because the structure of the space manipulator and its arm is very complexly and its volume tarnal small, we have to simplify the 3D model and focus on its quality of dispersion and support at both ends FIGURE IV, furthermore, we obtain the numbers of every constrained position $1 \sim 8$. Numbers 7,8 are the ones of the manipulator simplified. The different materials of different structure parameters are given by $[11,12]$. Hard aluminum alloy:

$$
\begin{aligned}
& \rho_{A l}=2.75 \mathrm{~g} / \mathrm{cm}^{3}=2.75 \times 10^{-9} \mathrm{t} / \mathrm{mm}^{3} \\
& E_{A l}=6.8 \times 10^{4} \mathrm{MPa}, v=0.3
\end{aligned}
$$

Steel:

$$
\begin{aligned}
& \rho_{g}=7.85 \mathrm{~g} / \mathrm{cm}^{3}=7.85 \times 10^{-9} \mathrm{t} / \mathrm{mm}^{3} \\
& E_{g}=2.1 \times 10^{5} \mathrm{MPa} \\
& v=0.3
\end{aligned}
$$




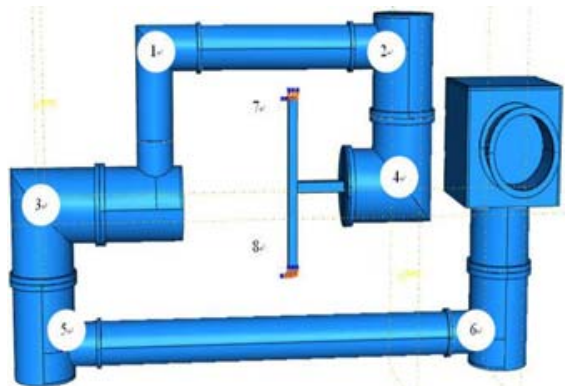

FIGURE III. 3D MECHANICAL AND CONSTRAINED POSITIONS

For the structure of the space manipulator and its arm, we discrete it and get 3-D body model which has n-degree of freedom, then it is easy to obtain n-degree of freedom linear invariant systems differential equations

$$
\mathrm{MX}+\mathrm{C} \dot{\mathrm{X}}+\mathrm{KX}=\mathrm{F}
$$

where, $\mathrm{M} 、 \mathrm{C} 、 \mathrm{~K}$ are the $\mathrm{n} \times \mathrm{n}$ mass matrix, damping matrix and stiffness matrix.

$$
\mathrm{X}=\left[\begin{array}{llll}
x_{1} & x_{2} & \cdots & x_{n}
\end{array}\right]^{T}, \mathrm{~F}=\left[\begin{array}{llll}
f_{1} & f_{2} & \cdots & f_{n}
\end{array}\right]^{T}
$$

where $\mathrm{X}$ is the displacement response, $\mathrm{F}$ is the external load excitation.

The eq. (1) transforms into the Fourier equation

$$
\left[\mathbf{K}-\mathbf{M} \omega^{2}+j \mathbf{C} \omega\right] \mathbf{x}(\omega)=\mathbf{F}(\omega)
$$

where $\omega$ and $\mathrm{j}$ are Laplace operator symbol, $\mathbf{Q}=\left\{q_{1}, q_{2}, \mathbb{\|} q_{N}\right\}^{T}$ is Modality matrix, $\mathrm{A}=\left[\mathrm{A}_{1}, \mathrm{~A}_{2}, \cdots, \mathrm{A}_{N}\right]^{T}$ stands for the influence factors of each order mode.

Because of the inherent frequency of the space manipulator and its arm system and its modes by the physical parameters of the system matrix $\mathrm{K}$ and $\mathrm{M}$ entirely, they are dose not matter with the external force $\mathrm{F}$. we get the dynamic equation of simplified system with n-degree freedom

$$
\mathrm{MX}+\mathrm{KX}=0
$$

The eq. (4) transforms into the Fourier equation

$$
\left(\mathrm{K}-\omega^{2} \mathrm{M}\right) \mathrm{A}=0
$$

We can also find out the flexibility matrix $\Delta$ and bring the flexibility matrix $\Delta$ into (5)

$$
\left(\Delta \mathrm{M}-\mathrm{I} / \omega^{2}\right) \mathrm{A}=0
$$

We calculate the low-level intrinsic modes of this space manipulator with the constrained positions 1 8 [13]. From the results of our calculation, the constrained points 7 and 8 of the space manipulator and the arm support end have different levels of vibration in the first eight modes, which are still quite powerful, such as: mode2, mode5, mode6 and the mode 7 . We must strengthen the constrained points 7 and 8 of the space manipulator and the arm support end. In addition, some departments of this space manipulator are vibration intense stress concentration, so we have to smooth by chamfer and avoid the stress concentration.

\section{OPTIMIZATION AND FINITE ELEMENT ANALYSIS}

When the constrained positions and the structure of this space manipulator have been identified, we want to get the dynamic states of them. But the different constrained states, the different stiffness matrix $\mathrm{k}$ or flexibility matrix and the different external incentives, the eq. (6) have different solutions. So we have to aim at every different state to modular Analysis. The work gives the program to ascertain the modes and vibrational shapes of each constrained state FIGURE IV.

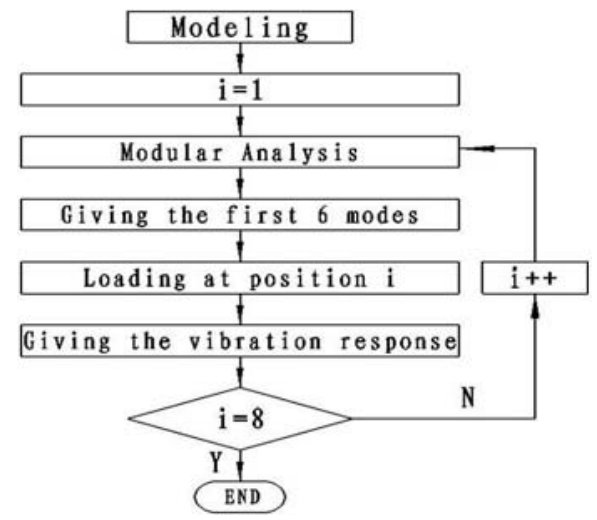

FIGURE IV. THE PROGRAM OF THE MODES AND VIBRATION SHAPES

In this program, the case $i$ means that the position $i$ is released and other 7 positions are constrained, $i=1 \cdots 8$. We get the first 6 modes and vibrational shapes of each i. Especially, the constrained position 6 always is vibrational under the each $i$ and every mode, it must be reinforced. The other hand, the part of $i$ is severe vibrational, we obtain the impact factor of every $i$ [14].

\section{GROUPING AND VERIFICATION}

The constrained points 7and 8 of the space manipulator have different levels of vibration based on front work. When the spacecraft flies, the constrained points 7 and 8 of the space manipulator might go off. They must bring the huge inertia impact force on the entire space manipulator and its arm after going off. We have to analyze mechanical characteristic at constrained place 4 and support end of the 3D mechanical model after going off. The 3D model is loaded respectively $1000 \mathrm{KN}$ on the places of 4 and support end. 
When the space manipulator and its arm are loaded external forces, we will obtain Amplitude values of the different frequencies in the different constrained states from the front of the work. Then we will get the fracture sequence of the constrained positions of the space manipulator and its arm by the program as shown in FIGURE V.

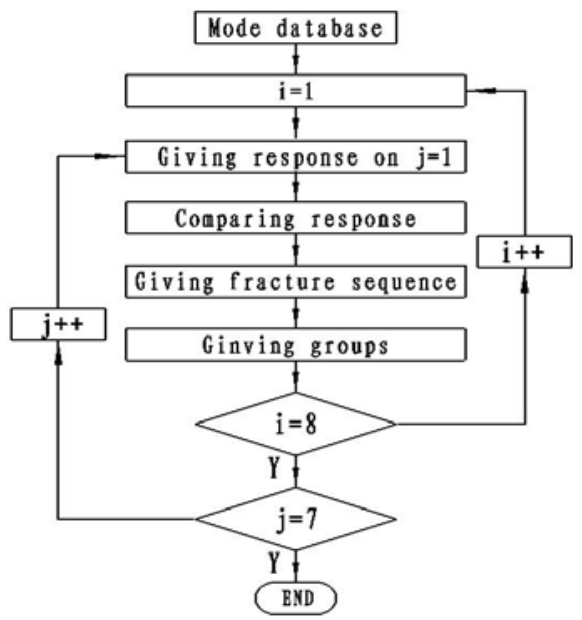

FIGURE V. THE GROUPING PROGRAM

In the program, $i=1,2,8$ and $j=1,2, \ldots 7$, grouping

$$
\begin{aligned}
& j=1, w \in[0,20) \mathrm{Hz} ; \\
& j=2, w \in[20,35) \mathrm{Hz} ; \\
& j=3, w \in[35,60) \mathrm{Hz} ; \\
& j=4, w \in[60,80) \mathrm{Hz} ; \\
& j=5, w \in[80,110) \mathrm{Hz} ; \\
& j=6, w \in[110,140) \mathrm{Hz} ; \\
& j=7, w \in[140,180) \mathrm{Hz} .
\end{aligned}
$$

The program runs results for

$$
\begin{aligned}
& j=1,6>5>1>2>8>7>3>4 ; \\
& j=2,6>5>4>1>8>3>7>2 ; \\
& j=3,5>3>2>6>1>8>7>4 ; \\
& j=4,5>1=2=3=4=6=7=8 ; \\
& j=5,5>4>1>2>7>8>3>6 ; \\
& j=6,6>2>7>8>1>4>3>5 ; \\
& j=7,5>4>3>1>7>8>6>2 .
\end{aligned}
$$

The program results give the fracture sequence of the constrained positions of the space manipulator and its arm, the best fracture sequence: 65128734, the next one: 65418372 and 53261874 and so on. We can also analyze several optimized results by finite element software Abaqus and then find out the response of the overall structure of the space manipulator and its arm under optimized and loaded external forces.
Aiming at the best fracture sequence: 65128734, $\mathrm{j}=1$, this work selects three constrained cases: 651, 6512 and 65128 , respectively, pluses the external forces and analyzes the response of the overall structure of the space manipulator and its arm, lastly we determine the optimal solution of the constrained number. Firstly we calculated the low order modes of the three cases and took the first 4 modes compared as shown in FIGURE VI VIII.

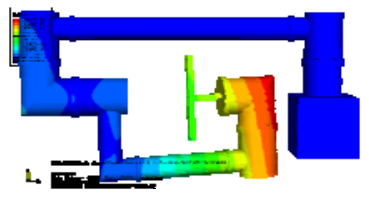

$\omega_{1}=6.732 \mathrm{~Hz}$

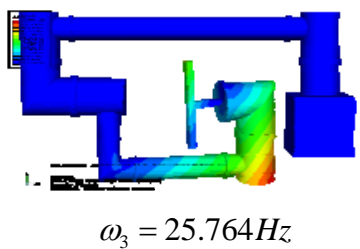

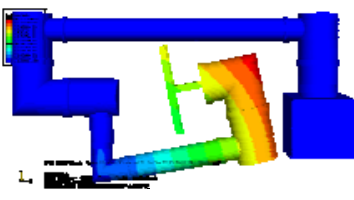

$\omega_{2}=12.220 \mathrm{~Hz}$

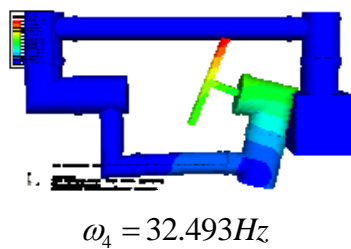

FIGURE VI. THE FIRST 4 MODES AND RESPONSE AFTER CONSTRAINING POSITIONS: 651

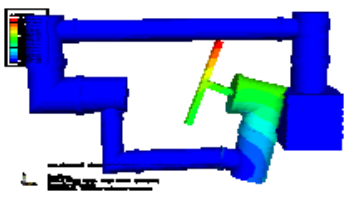

$\omega_{1}=19.867 \mathrm{~Hz}$

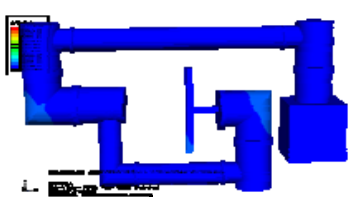

$\omega_{3}=46.153 \mathrm{~Hz}$

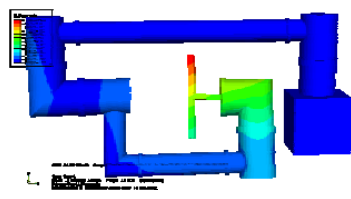

$\omega_{2}=32.477 \mathrm{~Hz}$

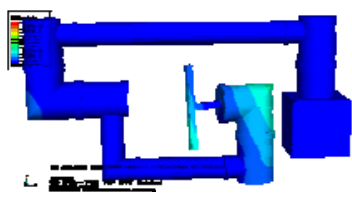

$\omega_{4}=61.801 \mathrm{~Hz}$
FIGURE VII. THE FIRST 4 MODES AND RESPONSE AFTER CONSTRAINING POSITIONS: 6512

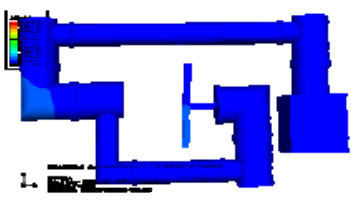

$\omega_{1}=46.193 \mathrm{~Hz}$

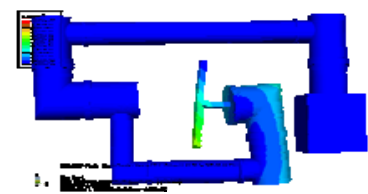

$\omega_{3}=71.268 \mathrm{~Hz}$

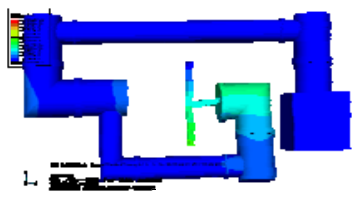

$\omega_{2}=61.146 \mathrm{~Hz}$

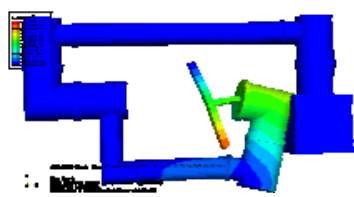

$\omega_{4}=86.901 \mathrm{~Hz}$
FIGURE VIII. THE FIRST 4 MODES AND RESPONSE AFTER CONSTRAINING POSITIONS: 65128 
From the results of our calculation, the cases 65128 and 6512 are similar, however, the case 651 is lager deformation. We choose the best case 6512 which has the appropriate constrained number finally. On this base, we also did the static analysis as shown in FIGURE IX.
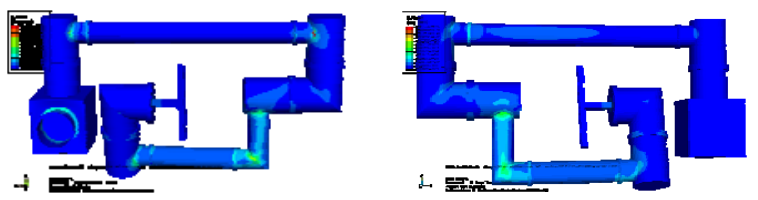

FIGURE IX. STATICS ANALYSIS AFTER CONSTRAINING POSITIONS: 6512

From the calculated results, it is large destroyed to the whole space manipulators and supporting arm after the constrained places gone off. We must do more in-depth static and dynamic analysis.

\section{CONCLUSIONS}

In this work, we design a kind of space manipulator and optimize all of constrained nodes, and then the static analysis is analyzed based on the calculation results. And last, the least number of constraint nodes are gotten. Hope that subsequent work can more considering structural materials of the influencing factors, and hope that the work can promote the industrial robot research and rapid development.

\section{ACKNOWLEDGMENT}

The authors would like to thank the referees for their valuable comments and suggestions that have improved the paper immeasurably. The authors are grateful to the support of the National Natural Science Foundation of China (Grant No.51508237); Natural Science Foundation of Jiangsu Province (Grant No.BK20140560); Research Foundation for Advanced Talents of Jiangsu University (Grant No.14JDG161; 14JDG162).

\section{REFERENCES}

[1] H. Huang, B. Li, Z. Deng, Y. Hu. A 6-DOF adaptive parallel manipulator with large tilting capacity. Robotics and ComputerIntegrated Manufacturing. 2012, Vol.28, pp. 275-283.

[2] L. Torabi, K. Gupta. An autonomous six-DOF eye-in-hand system for in situ 3D object modeling. International Journal of Robotics Research. 2012, Vol.31, pp. 82-100.

[3] Po-Yang Lin, Win-Bin Shieh, Dar-Zen Chen. Design of Statically Balanced Planar Articulated Manipulators With Spring Suspension. IEEE Transactions on Robotics.2012, Vol.28, pp. 12-21.

[4] K. Alexandr, F. Benoit, C. Stephane, etc. Identification of the manipulator stiffness model parameters in industrial environment. Mechanism and Machine Theory. 2015, Vol.90, pp. 1-22.

[5] T. Sun, J. Wu, L. Chen. Investigation of New Properties of Magnetorheological Fluids with Carbon Nanotubes. Journal of Computational and Theoretical Nanoscience. 2015(11), Vol.12:P41434146.

[6] G. B. Bhusan, S. B. Kumar, S. Rana. Realtime performance analysis of different combinations of fuzzy-PID and bias controllers for a two degree of freedom electrohydraulic parallel manipulator. ROBOTICS AND COMPUTER-INTEGRATED MANUFACTURING. 2015, Vol.34, pp. 62-69.
[7] P. Vinay, G. Kamal. A hierarchical and adaptive mobile manipulator planner with base pose uncertainty. AUTONOMOUS ROBOTS. 2015, Vol.39, pp. 65-85.

[8] B. Lee. Development of coaxial wire reduction gear for manipulator robots. ELECTRONICS LETTERS. 2015, Vol.51, pp. 747-748.

[9] M. Shimizu. Analytical inverse kinematics for 5-DOF humanoid manipulator under arbitrarily specified unconstrained orientation of endeffector. ROBOTICA. 2015, Vol.33, pp. 747-767.

[10] A. Klimchik, D. Chablat, A. Pashkevich. Static stability of manipulator configuration: Influence of the external loading. EUROPEAN JOURNAL OF MECHANICS A-SOLIDS. 2015, Vol.51, pp. 193-203.

[11] C. Escande, T. Chettibi, R.Merzouki, etc. Kinematic Calibration of a Multisection Bionic Manipulator. IEEE-ASME TRANSACTIONS ON MECHATRONICS. 2015, Vol.20, pp. 663-674.

[12] A. Rezaei, A. Akbarzadeh. Study on Jacobian, singularity and kinematics sensitivity of the FUM 3-PSP parallel manipulator. MECHANISM AND MACHINE THEORY. 2015, Vol.86, pp. 211-234.

[13] T. Sun, X. Peng, S. Jiao, C. Xu, J. Li. Simulation of Particle Dynamics of Nano-Magnetorheological Materials in External Magnetic Fields. Journal of Computational and Theoretical Nanoscience. 2014(6), Vol.11:P1518-1523.

[14] M. H. Korayem, A. M. Shafei. Motion equation of nonholonomic wheeled mobile robotic manipulator with revolute-prismatic joints using recursive Gibbs-Appell formulation. APPLIED MATHEMATICAL MODELLING. 2015, Vol. 39, pp. 1701-1716. 